\title{
Bio- and chemosensors based on the thermal boundary resistance at solid-liquid interfaces
}

\author{
Patrick Wagner ${ }^{1}$ \\ ${ }^{1}$ KULeuven, Department of Physics and Astronomy, Soft-Matter Physics and Biophysics Section, \\ Celestijnenlaan 200 D, 3001 Leuven, Belgium \\ PatrickHermann.Wagner@kuleuven.be
}

\begin{abstract}
:
This contribution will give an overview on a recently developed sensing principle, the Heat-Transfer Method HTM. In analogy with impedance spectroscopy that employs capacitive- or Faradaic electrical currents, HTM operates with thermal currents that pass the interface between a functionalized sensor chip and the supernatant liquid. The thermal resistance $R_{\text {th }}$ of the interface is highly sensitive to the conformation of the functional layer and/or to its binding state with target molecules. HTM applications till date include the characterization of mutations in DNA, the monitoring of phase transitions in lipids, and the selective and quantitative detection of neurotransmitters, proteins, cancer cells and bacteria. Furthermore, HTM can easily be combined with other sensing principles to gain orthogonal information on target molecules. Regarding detection limits, HTM is similar to non-Faradaic impedance spectroscopy (nanomolar range) with the difference that HTM can also operate with electrically insulating chip materials and insulating receptor coatings such as surface-imprinter polymer layers. The overview will conclude with recent observations on heat transfer at the molecular scale and will present an outlook to bio- and chemosensors that utilize thermal-wave techniques.
\end{abstract}

Key words: Bioanalysis, label-free sensors, impedance spectroscopy, thermal boundary resistance, molecularly- and surface-imprinted polymers.

\section{A First Example: DNA melting}

In heat-transfer experiments on molecular DNA brushes the probe DNA was immobilized covalently on synthetic sapphire chips by using silanization and the EDC coupling route. The probe DNA was hybridized with complementary and with mismatched target DNA corresponding to single-nucleotide polymorphisms. The melting temperature, $T_{m}$, is obtained by increasing the backside temperature of the chip, $T_{1}$, with a controlled heating power $P$ up to $90^{\circ} \mathrm{C}$. Measuring the temperature of the liquid, $T_{2}$, one obtains the thermal resistance $R_{\text {th }}$ via eq. (1):

$$
R_{t h}=\frac{T_{1}-T_{2}}{P}
$$

Figure 1 shows typical DNA-denaturation traces with a characteristic increase of $R_{t h}$ upon the transition from the double- (ds) to the singlestranded (ss) state. The melting temperature corresponds to the inflection points of the $R_{t h}\left(T_{1}\right)$ traces and the order of stability agrees with calculations based on the online HYTHER algorithm. For comparison, also data for a blank chip and a layer of linker molecules are shown.

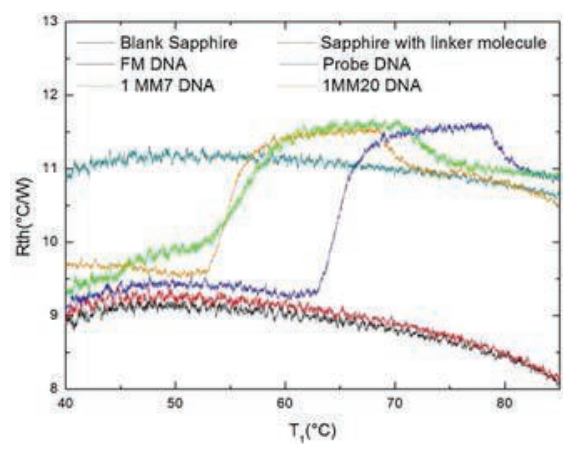

Fig. 1. Increase of $R_{\text {th }}$ upon thermal denaturation of complementary (cyan-colored line) and mismatched DNA duplexes (yellow and green lines) tethered to a synthetic sapphire chip [1].

The heat-flow blocking (increase of $R_{t h}$ ) is based on the different conformation: In the dsstate, the duplexes form rigid helices, oriented perpendicularly on the surface. In the ss-state the remaining fragments of probe DNA curl up into irregular structures (Flory spheres), which results in an increasing surface coverage of the chip with a soft-matter interlayer [2]. According to an XPS analysis, the areal density of probe DNA is in the order of $2 \cdot 10^{12}$ fragments per $\mathrm{cm}^{2}$. 


\section{Detection of Proteins and Neurotransmitters}

Figure 2 illustrates the layout of a sensor chip for protein detection, in this case the peanut allergen Ara h1 [3]. The receptors are aptamers that are coupled to gilded silicon chips via thiol linkers; a BSA blocking layer prevents nonspecific protein adsorption. The binding of Ara h1 leads to a concentration-dependent increase of $R_{t h}$ with a detection limit of $5 \mathrm{nM}$ in buffer liquids. For a proof-of-concept on food samples, we prepared an extract of peanut butter in TGK buffer and determined a concentration (26 nM) that agrees very well with the native concentration of this allergen in peanuts.

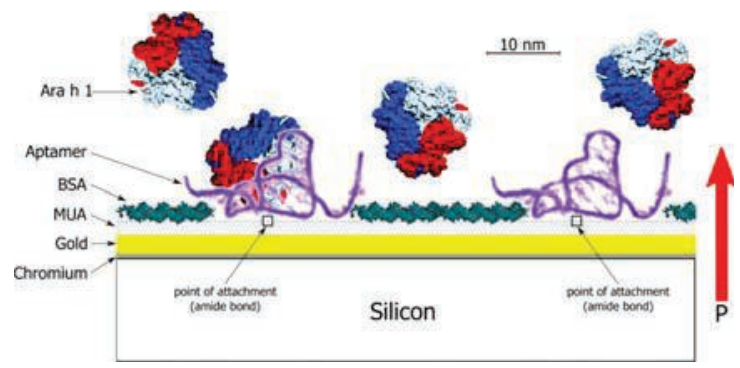

Fig. 2. Scheme of the bio-recognition layer for the detection of the peanut protein Ara h1 using aptamer receptors [3]. For HTM measurements, the backside temperature of the chip is kept constant at $37.0^{\circ} \mathrm{C}$. Binding of proteins results in a decrease of the thermal current $P$ that is indicated by an arrow.

Regarding the detection of small molecules such as neurotransmitters and inflammation markers (serotonin, histamine, nicotine), we used molecularly imprinted polymers (MIPs) as synthetic receptors. The MIP layers were either made of polymer micro-particles that were deposited onto aluminum chips by soft lithography or by RAFT polymerization on graphene-oxide flakes [4]. All MIPs were found selective with respect to their molecular targets as proven by reference tests with competitor molecules including cotinine and histidine. Similarly to the aptamer-protein case, also the MIP-coated sensor chips show a concentrationdependent increase of the heat-transfer resistance. We interpret this in terms of a blocking of the water-filled channels in the porous MIP materials upon binding of target molecules.

While the combination of HTM with MIP receptors reaches nanomolar detection limits for diluted body fluids, there is evidence that the concept still holds with whole-blood samples [5]. For this, a single-shot HTM device was designed as a first step towards fast serotonin detection in a point-of-care context. By using a non-functional reference channel, the $R_{\text {th }}$ data can be corrected for non-specific adsorption effects even in such complex matrices.

\section{HTM for Cancer Cells and Bacteria}

Also cells can be detected and identified using thermal currents thanks to the low thermal conductivity of their membranes. For this, we prepared whole-cell receptors using the surface-imprinting concept introduced by $F$. L. Dickert and coworkers; see e.g. ref. [6]. These imprints are generated by pressing template cells into semi-cured polyurethane layers. After hardening the polymer and removing the templates, the chips are covered with shallow imprints that can rebind a given cell type selectively due to size-, shape- and chemical complementarities. Fig. 3 shows exemplarily an imprint of an $E$. coli bacterium as visualized by atomic-force microscopy. These imprints can be regenerated for at least 10 times without losing their binding affinity. Using this approach, we could e.g. distinguish between different types of macrophages and human cancer-cell lines (leukemia vs. breast cancer) [7]. The current detection limit for bacteria is in the range of $10^{4}$ $\mathrm{CFU} / \mathrm{mL}$ and bacterial strains can be selectively identified even in presence of a 100-fold excess of competitor bacteria in the liquid [8].

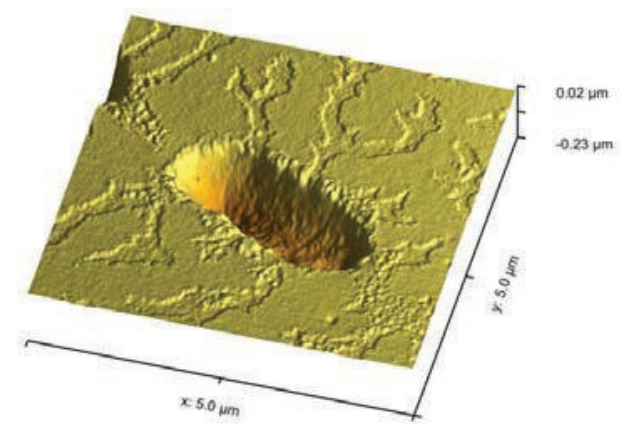

Fig. 3. AFM micrograph of a single E. coli imprint on a polyurethane-coated glass chip (unpublished). The imprint has a depth in the order of $500 \mathrm{~nm}$.

\section{References}

[1] M.S. Murib, W.S. Yeap et al., Sensors and Actuators B: Chemical 230, 260-271 (2016); doi: 10.1016/j.snb.2016.02.027

[2] B. van Grinsven et al., ACS Nano 6 (3), 2712-2721 (2012), doi: 10.1021/nn300147e

[3] M. Peeters, B. van Grinsven et al., ACS Applied Materials \& Interfaces 7, 10316-10323 (2015), doi: 10.1021/acsami.5b00994

[4] M. Peeters et al., Analytical and Bioanalytical Chemistry 405 (20), 6453-6460 (2013), doi: 10.1007/s00216-0137024-9; M. Peeters et al., Sensors and Actuators B: Chemical 203, 527-535 (2014), doi: 10.1007/s00216013-7024-9

[5] T. Vandenryt et al., Sensors 17 (12), art. no. 2701 (2017), doi: 10.3390/s17122701

[6] O. Hayden, K. J. Mann, S. Krassnig, F. L. Dickert, Angewandte Chemie - Intl. Edition 45 (16), 2626-2629 (2006), doi: 10.1002/anie.200502857

[7] K. Eersels, B. van Grinsven et al., ACS Applied Materials \& Interfaces 5, 7258-7267 (2013), doi: 10.1021/am401605d

[8] B. van Grinsven et al., ACS Sensors 1, 1140-1147 (2016), doi: 10.1021/acssensors.6b0043 